\title{
Établir une base de recherche en psychiatrie
}

$\overline{\mathrm{A}}$ notre époque de médecine factuelle, la rareté des données probantes de grande qualité en santé mentale est frappante. Souvent, les professionnels de la santé mentale n'arrivent pas à obtenir d'information utile pour appuyer leurs décisions cliniques, situation que le directeur des National Institutes of Mental Health des États-Unis a dépeint comme «le malheureux état actuel» de la psychiatrie «où trop d'études de recherche offrent peu de pertinence immédiate pour la pratique et trop peu de pratique repose sur des résultats de recherche» ${ }^{1}$. Cette faiblesse se répercute à la fois sur le traitement de la schizophrénie et du trouble bipolaire, qui touchent chacun environ I \% de la population (300 000 Canadiens), et de la dépression, qui touchera environ $8 \%$ des Canadiens au cours de leur vie. Quelles sont les répercussions de cette situation pour les patients?

Dans ce numéro, Deshauer et ses collaborateurs soulignent que les études sur les antidépresseurs sont habituellement trop courtes pour être signifiantes ${ }^{2}$. On a aussi reproché aux études portant sur des médicaments psychiatriques de minimiser l'importance des effets indésirables ou des risques d'abus des médicaments à l'étude ${ }^{3,4}$. En pédiatrie, il n'existe tout simplement pas d'études d'envergure portant sur des antipsychotiques atypiques ${ }^{5}$. En dépit de ce manque de données probantes, la grande majorité des pédiatres du développement et des pédopsychiatres du Canada prescrivent quand même ces médicaments ${ }^{6}$. Ce n'est guère mieux chez les adultes. Les antipsychotiques atypiques constituent un pilier du traitement de la schizophrénie, mais on a jugé peu fiables beaucoup d'études comparatives de ces médicaments : «Il semble que c'est l'entreprise qui commandite l'étude qui produit le meilleur médicament", écrivaient les auteurs d'une analyse effectuée en $2006^{7}$.

L'effort en cours vise toutefois à transformer la base de données probantes dans ce domaine. Pilotée par les National Institutes of Health des États-Unis qui s'inspirent de leurs succès antérieurs dans le cadre d'efforts semblables en cardiologie et en oncologie, la stratégie consiste à créer des réseaux de cliniciens dans de nombreux centres et à suivre des centaines de patients dans le contexte d'études définitives d'envergure ${ }^{8}$. Un réseau financé par les National Institutes of Health a ainsi évalué l'efficacité de traitements pharmacologiques contre la dépression au cours d'une étude de traitements séquencés possibles (étude $S T A R^{\star} D$ : Sequenced Treatment Alternatives to Relieve Depression), que Rush décrit dans le présent numéro ${ }^{9}$. Les réseaux des National Institutes of Health se penchent aussi sur le trouble bipolaire, la schizophrénie, la dépression chez les adolescents et la maladie d'Alzheimer ${ }^{10-16}$. Au Royaume-Uni, un réseau financé par l'Institut de recherche médicale Stanley étudie l'utilisation de thymorégulateurs contre le trouble bipolaire ${ }^{17}$ et un réseau du National Health Service s'est penché sur des pharma- cothérapies contre la schizophrénie ${ }^{18}$, tandis qu'un réseau de 50 centres commandités par l'industrie en Europe et en Israël a évalué le traitement aux antipsychotiques chez les jeunes qui en sont au premier stade de la schizophrénie ${ }^{19}$.

Des rapports de ces études "pragmatiques» ou "pratiques» ont à peine commencé à faire leur apparition depuis 2 ou 3 ans, mais ils pourraient entraîner des changements importants de la pratique. Des études portant sur le traitement de la schizophrénie réalisées par les National Institutes of Health et au Royaume-Uni (études CATIE sur les antipsychotiques cliniques [Clinical Antipsychotic Trials of Intervention Effectiveness] et étude CUtLASS sur le rapport coût-efficacité des antipsychotiques les plus récents [Cost Utility of the Latest Antipsychotic Drugs in Schizophrenia Study]) ont révélé que les nouveaux antipsychotiques atypiques offrent peu d'avantages par rapport à ceux de la première génération, résultats si percutants qu'un éditorial à ce sujet portait le titre "CATIE et CUtLASS : pouvons-nous accepter la vérité? $»^{20}$.

Nous nous attendons à ce que les psychiatres accueillent avec joie la "vérité» que fournissent ces études définitives, tout comme le même type d'études en cardiologie, dont beaucoup ont été réalisées au Canada, ont entraîné une amélioration des soins. D'importantes questions demeurent toutefois sans réponse. Les États-Unis, l'Europe et le Royaume-Uni ont montré la voie à suivre en se concentrant sur la comparaison de résultats cliniquement importants dans des contextes naturels à grande échelle. Le Canada devrait participer à l'exercice, et même le diriger dans les domaines où nos chercheurs ont établi leur savoir-faire. Sans une impulsion puissante, les grandes études randomisées ne deviendront pas une priorité en psychiatrie. Par où faut-il donc commencer?

La Commission canadienne de la santé mentale, lancée en août 2007 sous la direction du sénateur Michael Kirby, a reçu IIo millions de dollars dans le budget fédéral de 2008. La commission devrait jouer un rôle crucial en attirant l'attention sur le déficit des connaissances et en demandant aux chercheurs de s'y attaquer. Une autre première étape consisterait pour les Instituts de recherche en santé du Canada (IRSC) de relever les défis posés par l'établissement de réseaux de cliniciens capables de réaliser des études d'envergure en psychiatrie. Le $\mathrm{D}^{\mathrm{r}}$ Alain Beaudet, qui accédera à la présidence des IRSC en juillet 2008, pourrait très bien considérer qu'un leadership scientifique à l'échelle nationale s'impose pour lancer de tels études. Aux États-Unis, le chef des National Institutes of Health a intégré les études d'envergure dans le plan directeur des instituts et le chef du National Institute of Mental Health a préconisé ces études comme moyen de refermer l'écart entre la recherche et la pratique en psychiatrie ${ }^{1,21}$. Les chercheurs canadiens en psychiatrie méritent une inspiration et un soutien semblables sous forme d'engagements financiers clairs. 
Dans l'état actuel de données probantes limitées ou de manque de données, les professionnels de la santé mentale ne peuvent que choisir au jugé les meilleurs traitements. Pour les patients atteints de troubles mentaux, cela signifie que leurs médecins ne savent pas pour quelle personne les traitements devraient donner des résultats ou échouer. La psychiatrie doit intégrer la recherche aux soins en réalisant des études d'envergure et en appliquant les résultats. Pour la santé mentale autant que pour les maladies physiques, nous devons offrir ce que nous avons de mieux sans avoir à jouer les devins.

\section{Miriam Shuchman MD}

Professeure agrégée de psychiatrie

Université de Toronto

Hôpital Women's College

Toronto (Ont.)

\section{Paul C. Hébert MD MHSc}

Rédacteur en chef

\section{$J A M C$}

Avec l'équipe de rédaction de l'éditorial (Rajendra Kale MD, Barbara Sibbald BJ, Ken Flegel MDCM MSc et Noni MacDonald MD MSc)

Intérêts concurrents : aucuns dans le cas de Miriam Shuchman. Aucuns déclarés pour Paul Hébert en ce qui concerne cet éditorial; pour plus de renseignements, veuillez consulter http://www.cmaj.ca/misc/edboard.shtml. Voir www.cmaj.ca/misc/edboard.shtml pour les déclarations de l'équipe de rédaction de l'éditorial.

\section{RÉFÉRENCES}

I. Insel TR. Beyond efficacy: the STAR ${ }^{\star} \mathrm{D}$ trial. Am J Psychiatry 2006;I63:5-7.

2. Deshauer D, Moher D, Fergusson D, et al. Selective serotonin reuptake inhibitors for unipolar depression: a systematic review of classic long-term randomized con- trolled trials. JAMC 2008;178:1293-301

3. Kruszewski SP. Euphorigenic and abusive properties of modafinil. Am J Psychiatry 2006;163:549.

4. Kruszewski SP, Klotz SG. Effects of topiramate. Am J Psychiatry 2007;164:526-7.

5. McClellan JM. Olanzapine and pediatric bipolar disorder: evidence for efficacy and safety concerns. Am J Psychiatry 2007; I64:I462-4.

6. Doey T, Handelman K, Seabrook JA, et al. Survey of atypical antipsychotic prescribing by Canadian child psychiatrists and developmental pediatricians for patients aged under 18 years. $R$ Can Psychiatrie 2007;52:363-8.

7. Heres S, Davis J, Maino K, et al. Why olanzapine beats risperidone, risperidone beats quetiapine, and quetiapine beats olanzapine: an exploratory analysis of headto-head comparison studies of second-generation antipsychotics. Am J Psychiatry 2006; $163: 185-94$.

8. March JS, Silva SG, Compton S, et al. The case for practical clinical trials in psychiatry. Am J Psychiatry 2005;162:836-46.

9. Rush AJ. Developing the evidence for evidence-based practice. JAMC 2008;178: I3I -5

Io. Lieberman JA, Stroup TS, McEvoy JP, et al. Effectiveness of antipsychotic drugs in patients with chronic schizophrenia. N Engl J Med 2005;353:1209-23.

II. Rosenheck RA, Leslie DL, Sindelar J, et al. Cost-effectiveness of second generation antipsychotics and perphenazine in a randomized trial of treatment for chronic schizophrenia. Am J Psychiatry 2006;163:2080-9.

I2. Schneider LS, Tariot PN, Dagerman KS, et al. Effectiveness of atypical antipsychotic drugs in patients with Alzheimer's disease. N Engl J Med 2006;355:1525-38.

I3. Rush AJ, Trivedi MH, Wisniewski SR, et al. Acute and longer-term outcomes in depressed outpatients who required one or several treatment steps: a STAR ${ }^{\star} \mathrm{D}$ report. Am J Psychiatry 2006;163:1905-17.

I4. Sachs GS, Nierenberg AA, Calabrese JR, et al. Effectiveness of adjunctive antidepressant treatment for bipolar depression. N Engl J Med 2007;356:I7II-22.

I5. March JS, Silva S, Petrycki S, et al. The Treatment for Adolescents with Depression Study (TADS): long-term effectiveness and safety outcomes. Arch Gen Psychiatry 2007;64:II32-43.

I6. Brent D, Emslie G, Clark G, et al. Switching to another SSRI or to venlafaxine with or without cognitive behavioral therapy for adolescents with SSRI-resistant depression: the TORDIA randomized controlled trial. JAMA 2008;299:90I-I3.

I7. Geddes J, Goodwin G, Rendell J, et al. New trial should clarify lithium use in bipolar disorder. $B M J$ 2002;325:44I.

I8. Jones PB, Barnes TR, Davies L, et al. Randomized controlled trial of the effect on quality of life of second- vs first-generation antipsychotic drugs in schizophrenia: Cost Utility of the Latest Antipsychotic Drugs in Schizophrenia Study (CUtLASS I). Arch Gen Psychiatry 2006;63:1079-87.

I9. Kahn RS, Fleischhacker WW, Boter $\mathrm{H}$, et al. Effectiveness of antipsychotic drugs in first-episode schizophrenia and schizophreniform disorder: an open randomized clinical trial. Lancet 2008;371:1085-97.

20. Lewis S, Lieberman J. CATIE and CUtLASS: can we handle the truth? BrJ Psychiatry 2008;I92:I6I-3.

2I. Zerhouni E. The NIH Roadmap. Science 2003;302:63-72. 